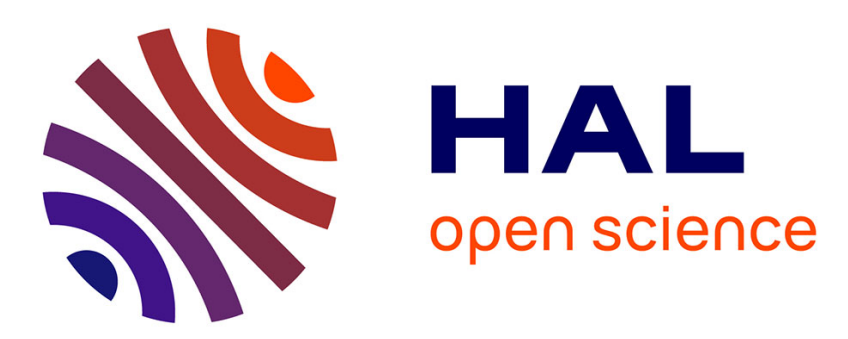

\title{
Une approche non destructive pour l'identification de contraintes de contact
}

\author{
J. Ben Abdallah, Marc Bonnet
}

\section{To cite this version:}

J. Ben Abdallah, Marc Bonnet. Une approche non destructive pour l'identification de contraintes de contact. Comptes Rendus de l'Academie des Sciences Serie II, 2000, 328, pp.525-529. 10.1016/S16207742(00)00022-2 . hal-00092384

\section{HAL Id: hal-00092384 \\ https://hal.science/hal-00092384}

Submitted on 11 Aug 2008

HAL is a multi-disciplinary open access archive for the deposit and dissemination of scientific research documents, whether they are published or not. The documents may come from teaching and research institutions in France or abroad, or from public or private research centers.
L'archive ouverte pluridisciplinaire HAL, est destinée au dépôt et à la diffusion de documents scientifiques de niveau recherche, publiés ou non, émanant des établissements d'enseignement et de recherche français ou étrangers, des laboratoires publics ou privés. 


\title{
Une approche non destructive pour l'identification de contraintes de contact
}

\author{
Jalel Ben Abdallah $^{\text {a,b }}$, Marc Bonnet ${ }^{\mathrm{c}}$
}

a Ecole Supérieure d'Ingenieurs de l'Equipement Rural (ESIER). Route du Kef, Km 5. Medjez-el-bab, Tunisie.

b Laboratoire de modélisation mathématique et numérique dans les sciences de l'ingenieur (LAMSIN), Ecole Nationale d'Ingénieurs de Tunis - BP 37, 1002 Tunis-Belvédère.

Courriel : jalel.benabdallah@enit.rnu.tn

c Laboratoire de Mécanique des Solides (CNRS UMR 7649), Ecole Polytechnique - 91128 Palaiseau cedex Courriel : bonnet@lms.polytechnique.fr

(Reçu le , accepté le )

Résumé. Cette Note concerne une méthode non destructive d'identification de la distribution de contraintes de contact entre un poinçon rigide et un massif élastique semi-infini au moyen de mesures du déplacement normal et des déformations tangentielles à la surface libre du massif. Les résultats numériques, obtenus pour des configurations axisymétriques, valident cette méthode, y compris en présence de données expérimentales inexactes et quand la zone de contact n'est pas a priori exactement connue (expérience d'indentation). (C) 2000 Académie des sciences/Éditions scientifiques et médicales Elsevier SAS

identification non destructive /contact élastique / problème inverse / essai d'indentation

\section{A Non-destructive Approach for the Identification of Contact Stresses}

Abstract. This note presents a non destructive method for the identification of contact stresses between a rigid punch and an elastic half-space from measurements of normal displacements and tangential strains on the traction-free part of the surface. Numerical results obtained on axisymmetric sample problems validate the identification method, even in the presence of imperfect data and when the contact zone is not exactly known a priori. (c) 2000 Académie des sciences/Éditions scientifiques et médicales Elsevier SAS

non destructive identification / elastic contact / inverse problem / indentation test

\section{Abridged English version}

This Note addresses the identification of contact stress distributions $p_{i}\left(y_{1}, y_{2}\right)(i=1,2,3)$ between a rigid punch and an elastic half-space (defined by $\left\{x_{3} \leq 0\right\}$ in terms of adequate Cartesian coordinates $\left.\left(O x_{1} x_{2} x_{3}\right)\right)$ using measurements of normal displacements $u_{3}\left(x_{1}, x_{2}\right)$ and in-plane strains $\varepsilon_{k \ell}\left(x_{1}, x_{2}\right)$ $(k, \ell=1,2)$ at sensor locations $\boldsymbol{x}$ away from the punch. Analysis of the reconstructed contact stress distributions is expected to provide valuable insight about the nature and characteristics of the friction law.

Note présentée par Everiste SANCHEZ-PALEnCIA

S1620-7742(01)0????-?/FLA

(c) 2000 Académie des sciences/Éditions scientifiques et médicales Elsevier SAS. Tous droits réservés. 


\section{J. Ben Abdallah, M. Bonnet}

Well-known integral representation formulas allow to recast the identification problem in the form of a system of first-kind Fredholm integral equations (1, 2, 2), which constitute a linear ill-posed problem [4] $\left(U_{i}^{k}\right.$ : components of the Boussinesq $(k=3)$ and Cerruti $(k=1,2)$ fundamental solutions; $S$ : contact zone under the punch). Eqs. (1, 22) are valid for a contact zone of arbitrary shape, but the present study is restricted to axisymmetric conditions about $O x_{3}$ (so that $S$ is a disk of radius $a$ ). Using cylindrical coordinates $\left(y_{1}=\rho \cos \theta, y_{2}=\rho \sin \theta\right)$, an analytical angular integration reduces (11) to (3, (4) [5], from which expressions for $\varepsilon_{\rho \rho}(r)=\mathrm{d} u_{\rho} / \mathrm{d} r$ and $\varepsilon_{\theta \theta}(r)=u_{\rho} / \rho$ are easily derived; one thus obtains a system of first-kind integral equations of the form (5).

In practice, $\varepsilon_{\rho \rho}, \varepsilon_{\theta \theta}, u_{3}$ are only known at discrete observation points $r_{1}, \ldots, r_{m}$. The unknowns $p_{\rho}, p_{3}$ must also be interpolated, here using continuous and piecewise linear functions on $0 \leq \rho \leq \hat{a}$ ( $\hat{a}$ : an upper bound of $a$ if $a$ is not known exactly). Hence, from $(\$)$, the $(2 n-1)$-vector $\boldsymbol{p}=\left\{p_{3}^{1}, p_{\rho}^{2}, p_{3}^{2}, \ldots, p_{\rho}^{n}, p_{3}^{n}\right\}$ of unknowns and the $3 m$-vector $\boldsymbol{d}=\left\{\varepsilon_{\rho \rho}\left(r_{i}\right), \varepsilon_{\theta \theta}\left(r_{i}\right), u_{3}\left(r_{i}\right), 1 \leq i \leq m\right\}^{T}$ of observations satisfy $\boldsymbol{G} \boldsymbol{p}=\boldsymbol{d}$, where the matrix $G$ is found to be very ill-conditioned. Hence a Tikhonov-type regularized formulation (6) is used [7], the regularization parameter $\alpha$ being computed using the cross-validation method [3, 4].

The inversion method is numerically tested for a Hertzian load $(a=15 \mathrm{~mm})$ and a Coulomb friction law of coefficient $f=0.45$, for which simulated data is computed using (5). Numerical inversions are made for $n=20$ and $m=7$ or 70 (the $r_{i}$ are regularly spaced, with $20 \mathrm{~mm} \leq r \leq 50 \mathrm{~mm}$ ). Simulated Gaussian random noise with zero mean and relative standard deviation $e$ is added to the simulated data $(e=0,0.01$ or 0.03). Using first $\hat{a}=a$ (i.e. assuming a known contact radius), inversion results for $m=7$ are fair with $e=0$ but become poor in the presence of data noise; results for $m=70$ are better (figure 1). Then, using only the upper bound $\hat{a}^{2}=\delta(2 R-\delta)$ of $a$ ( $\delta$ : indentation depth, $R$ : punch radius), inversion results for both $m=7$ and $m=70$ tend to oversmooth the solution and not to reflect very accurately the contact and adherence radii (figure 2).

To achieve more accurate reconstruction of contact stress distributions under the punch for indentation experiments, the inversion approach should be refined. The next step will consist of treating the contact radius as unknown (the inversion being non-linear with respect to $a$ ). More sophisticated regularization approaches, aiming at avoiding oscillating reconstructions while allowing for limited amounts of discontinuities [6], may also be considered in the future.

\section{Introduction. Problèmes direct et inverse}

On s'intéresse dans cette Note à l'identification de distributions de contraintes de contact entre un poinçon rigide et un massif élastique semi-infini de caractéristiques élastiques connues. La connaissance de ces contraintes permettrait en particulier d'identifier la nature (locale ou non locale), le type (Coulomb, Tresca...) et les paramètres de la loi de frottement [2]. Ces contraintes ne sont toutefois pas directement accessibles à la mesure, la mise en place de capteurs sous le poinçon étant généralement impossible. Leur identification nécessite alors l'exploitation de mesures extérieures, et devient un problème inverse. On se propose ici d'examiner l'exploitation de mesures de déplacement normal et de déformations tangentielles à l'extérieur de la zone de contact.

Le problème direct consiste donc à calculer les déplacements et les déformations à la surface d'un massif élastique semi-infini, de comportement élastique homogène et isotrope, connaissant la distribution des contraintes normales et tangentielles dans la zone de contact $S$. Un repère cartésien $\left(O x_{1} x_{2} x_{3}\right)$ est choisi de telle sorte que le massif occupe le domaine $\left\{x_{3} \leq 0\right\}$ de frontière $\Gamma=\left\{x_{3}=0\right\}$; $\boldsymbol{e}_{3}$ en est donc la normale unitaire sortante. Les composantes $u_{k}\left(x_{1}, x_{2}\right)$ du déplacement en un point $\boldsymbol{x}=\left(x_{1}, x_{2}, 0\right)$ de la surface sont explicitement reliées à la distribution du vecteur-contrainte dans la zone de contact (composantes $\left.p_{i}\left(y_{1}, y_{2}\right)=\sigma_{i 3}\left(y_{1}, y_{2}\right)\right)$ par la formule de représentation intégrale [1] :

$$
u_{k}\left(x_{1}, x_{2}\right)=\int_{S} p_{i}\left(y_{1}, y_{2}\right) U_{i}^{k}\left(y_{1}-x_{1}, y_{2}-x_{2}\right) \mathrm{d} y_{1} \mathrm{~d} y_{2} \quad i, k \in\{1,2,3\}
$$


où $U_{i}^{k}\left(z_{1}, z_{2}\right)$ désigne la composante $i$ du champ de déplacement au point $\boldsymbol{z}$ d'un massif semi-infini à surface libre par l'application au point $O$ d'une force ponctuelle unitaire dirigée selon $x_{k}$ (solutions fondamentales de Boussinesq pour $k=3$ et de Cerruti pour $k=1,2$ ). Les composantes tangentielles du tenseur des déformations $\varepsilon(\boldsymbol{x})$ à la surface sont alors données par :

$$
\begin{array}{r}
\varepsilon_{k \ell}\left(x_{1}, x_{2}\right)=-\int_{S} p_{i}\left(y_{1}, y_{2}\right)\left[U_{i, \ell}^{k}\left(y_{1}-x_{1}, y_{2}-x_{2}\right)+U_{i, k}^{\ell}\left(y_{1}-x_{1}, y_{2}-x_{2}\right)\right] \mathrm{d} y_{1} \mathrm{~d} y_{2} \\
\quad(i \in\{1,2,3\}, k, \ell \in\{1,2\})
\end{array}
$$

Le problème inverse objet de cette Note consiste à reconstruire la distribution d'efforts $\boldsymbol{p}\left(y_{1}, y_{2}\right)$ sur $S$ à partir de mesures de déplacement et de déformations en des points $\boldsymbol{x}$ situés à l'extérieur de la zone de contact, en exploitant les identités (1), (2). Dans ces conditions, les noyaux $U_{i}^{k}\left(y_{1}-x_{1}, y_{2}-x_{2}\right)$ et $U_{i, k}^{\ell}\left(y_{1}-x_{1}, y_{2}-x_{2}\right)$ sont de carré intégrable sur $S$. Les relations (11), (1) définissent un problème d'inversion linéaire mal posé [4] (résolution d'équations intégrales de Fredholm de première espèce), d'un type général rencontré dans une grande variété de problèmes inverses mais dont l'utilisation en mécanique du contact ne nous semble pas encore avoir été faite.

\section{Mise en ouvre dans le cas axisymétrique}

Les équations (1), (2) sont applicables à des situations tridimensionnelles, mais on se restreint ici à l'hypothèse d'axisymétrie autour de l'axe $O x_{3}$; en particulier la zone de contact $S$ est supposée circulaire de rayon $a$. Après introduction de coordonnées cylindriques $\left(y_{1}=\rho \cos \theta, y_{2}=\rho \sin \theta, y_{3}=z\right)$ et une intégration analytique par rapport à $\theta \in[0,2 \pi]$, la formule (11) se réduit à [5] :

$$
\begin{aligned}
& u_{\rho}(r)=\frac{2(1-\nu)}{\pi \mu} \int_{0}^{a}\left\{\frac{t^{2}+r^{2}}{2 r(t+r)} K(k)-\frac{t+r}{2 r} E(k)\right\} p_{\rho}(t) \mathrm{d} t-\frac{(1-2 \nu)}{2 \mu r} \int_{0}^{a} t p_{3}(t) \mathrm{d} t \\
& u_{3}(r)=\frac{2(1-\nu)}{\pi \mu} \int_{0}^{a} \frac{t}{t+r} K(k) p_{3}(t) \mathrm{d} t
\end{aligned}
$$

où $r>a$ est la distance du point d'observation au centre de la zone de contact et $K(k), E(k)$ sont les intégrales elliptiques complètes de première et seconde espèce (avec $k^{2}=4 t r /(t+r)^{2}$ ). Les déformations $\varepsilon_{\rho \rho}(r)=\mathrm{d} u_{\rho} / \mathrm{d} r$ et $\varepsilon_{\theta \theta}(r)=u_{\rho} / \rho$ étant facilement obtenues à partir de (3), on a donc un système d'équations intégrales de première espèce de la forme :

$$
\left\{\begin{array}{l}
\varepsilon_{\rho \rho}(r) \\
\varepsilon_{\theta \theta}(r) \\
u_{3}(r)
\end{array}\right\}=\int_{0}^{a}\left[\begin{array}{ll}
g_{\rho \rho}(r, t) & g_{\rho 3}(r, t) \\
g_{\theta \rho}(\theta, t) & g_{\theta 3}(r, t) \\
g_{3 \rho}(r, t) & g_{33}(r, t)
\end{array}\right]\left\{\begin{array}{l}
p_{\rho}(t) \\
p_{3}(t)
\end{array}\right\} \mathrm{d} t
$$

Pour les applications, il faut supposer que $\varepsilon_{\rho \rho}, \varepsilon_{\theta \theta}, u_{3}$ sont mesurés en un nombre fini de points d'observation $r_{1}, \ldots, r_{m}$, et discrétiser les fonctions inconnues $p_{\rho}, p_{3}$. Les résultats d'inversion présentés ciaprès reposent sur une interpolation linéaire par morceaux et continue de $p_{\rho}$ et $p_{3}$, le segment $[0, \hat{a}]$ étant découpé en $n$ intervalles de longueurs égales ( $\hat{a}$ est un majorant du rayon de contact $a$, ce dernier n'étant pas nécessairement connu). En introduisant le $3 m$-vecteur $\boldsymbol{d}=\left\{\varepsilon_{\rho \rho}\left(r_{i}\right), \varepsilon_{\theta \theta}\left(r_{i}\right), u_{3}\left(r_{i}\right), 1 \leq i \leq m\right\}^{T}$ des observables et le $(2 n-1)$-vecteur $\boldsymbol{p}=\left\{p_{3}^{1}, p_{\rho}^{2}, p_{3}^{2}, \ldots, p_{\rho}^{n}, p_{3}^{n}\right\}$ des contraintes nodales inconnues (ayant posé $p_{\rho, 3}^{i}=p_{\rho, 3}((i-1) \hat{a} / n)$, imposé $p_{\rho, 3}(\hat{a})=0$ et remarqué que nécessairement $\left.p_{\rho}(0)=0\right)$, la relation (5) et la connaissance de la force de contact résultante conduisent à une relation matricielle de la forme $\boldsymbol{G} \boldsymbol{p}=\boldsymbol{d}$.

La matrice $\boldsymbol{G}$ est, comme attendu, très mal conditionnée : on a observé $\operatorname{Cond}(\boldsymbol{G}) \approx 610^{-16}$ ou $210^{-18}$ pour $(m, n)=(7,20)$ et $(70,20)$ respectivement, et la valeur singulière de $\boldsymbol{G}$ de $\operatorname{rang} k$ se comporte approximativement comme $\exp (-k)$. La résolution directe de $\boldsymbol{G} \boldsymbol{p}=\boldsymbol{d}$ étant impraticable, on introduit, suivant 


\section{J. Ben Abdallah, M. Bonnet}
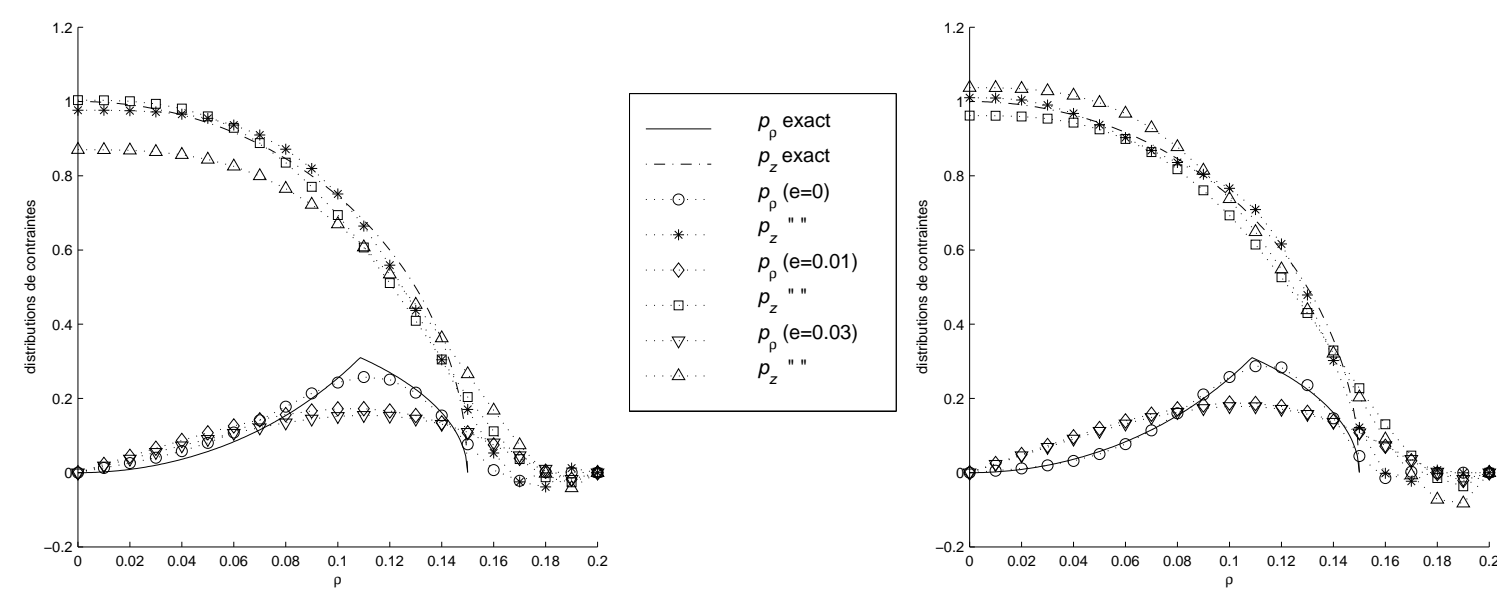

FIG. 1 - Identification de contraintes de contact hertziennes avec frottement de Coulomb : rayon de contact estimé par excès, 7 points de mesure (à gauche) et 70 points de mesure (à droite).

Identification of Hertzian contact stresses with Coulomb friction : upper-bound estimation of the contact radius, 7 measurement points (left), 70 measurement points (right).

une démarche classique [7], le problème régularisé :

$$
\boldsymbol{p}_{\alpha}=\arg \min _{\boldsymbol{q}} R_{\alpha}(\boldsymbol{q}) \quad R_{\alpha}(\boldsymbol{q})=\frac{1}{2}\left[(\boldsymbol{G} \boldsymbol{q}-\boldsymbol{d})^{T}(\boldsymbol{G} \boldsymbol{q}-\boldsymbol{d})+\alpha \boldsymbol{q}^{T} \boldsymbol{D} \boldsymbol{q}\right]
$$

Le paramètre de régularisation $\alpha$ est déterminé par un procédé itératif (critère de validation croisée [3]), et la matrice (positive et symétrique) $\boldsymbol{D}$, choisie dans le but de pénaliser les solutions $\boldsymbol{p}$ très oscillantes, provient de la discrétisation de

$$
\int_{0}^{\hat{a}}\left(\frac{d}{d t} p_{\rho}(t)\right)^{2} \mathrm{~d} t+\int_{0}^{\hat{a}}\left(\frac{d}{d t} p_{3}(t)\right)^{2} \mathrm{~d} t
$$

\section{Résultats et discussion}

Afin de tester la méthode d'inversion, on cherche à reconstruire un chargement hertzien, pour une zone de contact de rayon $a=15 \mathrm{~mm}$ et une loi de frottement de Coulomb $\left(p_{\rho}<f p_{3}\right.$ dans la zone d'adhérence, $p_{\rho}=f p_{3}$ dans la zone de glissement) de coefficient $f=0.45$;les données du problème inverse sont simulées par introduction de ce chargement dans (5), avec une subdivision $n=200$. Les $m$ points de mesure sont régulièrement répartis sur l'intervalle $20 \mathrm{~mm} \leq r \leq 50 \mathrm{~mm}$. Les données sont parfois bruitées par l'addition de nombres aléatoires gaussiens, de moyenne nulle et d'écart-type relatif $e=0,01$ ou 0,03 . Les inversions reposent sur une subdivision $n=20$.

Le rayon de contact est d'abord supposé connu $(\hat{a}=a)$. Les résultats obtenus par la procédure d'inversion régularisée pour $m=7$ sont convenables en l'absence de bruit mais médiocres sinon; ceux obtenus pour $m=70$ sont nettement meilleurs (figure 1).

On considère ensuite le cas où le rayon de contact $a$ n'est pas connu exactement mais estimé par excès à partir de la profondeur d'indentation $\delta$, supposée mesurée ainsi que l'effort normal $P\left(\hat{a}^{2}=\delta(2 R-\delta)\right.$ pour un poinçon sphérique de rayon $R$ ). Les résultats d'inversion obtenus pour 7 ou 70 points de mesure (figure 2) présentent des profils de contraintes convenables. On remarque cependant que, du fait de la régularisation adoptée qui favorise des profils très lisses, les rayons de contact et, surtout, de séparation 

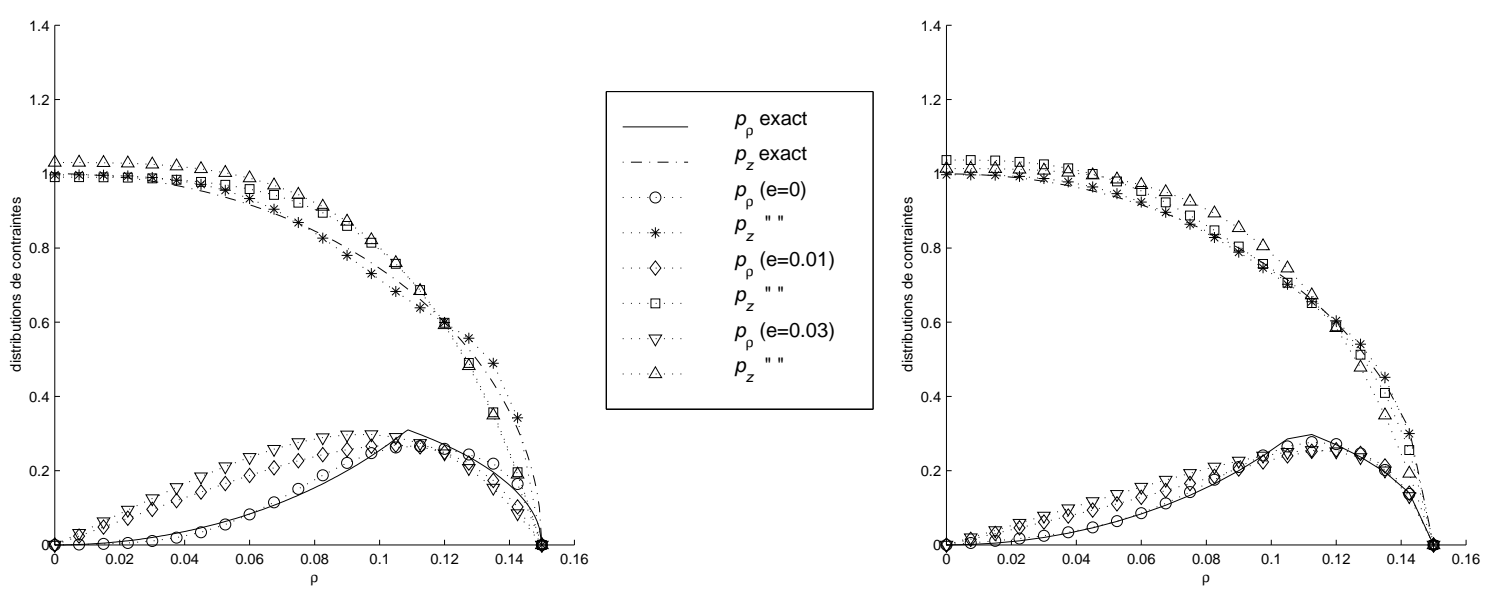

FIG. 2 - Identification de contraintes de contact hertziennes avec frottement de Coulomb : rayon de contact connu exactement $(\hat{a}=a), 7$ points de mesure (à gauche) et 70 points de mesure (à droite).

Identification of Hertzian contact stresses with Coulomb friction contact radius known $(\hat{a}=a): 7$ measurement points (left), 70 measurement points (right).

entre zones d'adhérence et de glissement, qui correspondent à des discontinuités de dérivée, n'apparaissent pas clairement si les données sont bruitées.

Dans l'optique d'une reconstruction plus précise des distributions de contraintes de contact sous le poinçon durant une expérience d'indentation (qui permettrait de déterminer l'existence d'une loi de frottement et, dans l'affirmative, d'en identifier les paramètres) il importe donc d'affiner la méthode d'inversion. Notre prochaine étape sera de traiter le rayon de contact $a$ comme une des inconnues, et de discrétiser $p_{\rho}, p_{3}$ sur l'intervalle variable $0 \leq r \leq a$; le problème d'inversion est alors bien entendu non-linéaire par rapport à $a$. On peut également envisager l'utilisation de techniques de régularisation plus sophistiquées, issues de la communauté du traitement du signal et de l'image [6] et conçues pour éviter les reconstructions oscillantes tout en permettant l'apparition d'un nombre limité de discontinuités.

\section{Références bibliographiques}

[1] Bonnet, M. Equations intégrales et éléments de frontière. CNRS Editions / Eyrolles, Paris, France (1995).

[2] BUI, H. D. Introduction aux problèmes inverses en mécanique des matériaux. Eyrolles, Paris (1993).

[3] Golub, G. H., Van Loan, C. F. Matrix computations (second edition). Johns Hopkins University Press, Baltimore (1989).

[4] HAnsen, P. C. Rank-deficient and discrete ill-posed problems. SIAM, Philadelphia, USA (1998).

[5] Johnson, K. L. Contact mechanics. Cambridge University Press (1985).

[6] MARTIN, TH. Inversion bayésienne du problème non-linéaire de tomographie d'impédance électrique modélisé par une méthode d'éléments finis. Thèse de Doctorat, Université Paris XI Orsay, France (1997).

[7] Tikhonov, A. N., Arsenin, V. Y. Solutions to ill-posed problems. Winston-Wiley, New York (1977). 